\title{
Image-enhanced bronchoscopic evaluation of bronchial mucosal microvasculature in COPD
}

This article was published in the following Dove Press journal: International Journal of COPD

29 September 2016

Number of times this article has been viewed

\author{
Eman Mahmoud Fathy' \\ Hanaa Shafiek' \\ Tamer S Morsi ${ }^{1, \dagger}$ \\ Bassma El Sabaa ${ }^{2}$ \\ Abdelaziz Elnekidy ${ }^{3}$ \\ Mohamed Elhoffy' \\ Mohamed Samy Atta' \\ 'Chest Diseases Department, \\ ${ }^{2}$ Pathology Department, ${ }^{3}$ Radiology \\ Department, Faculty of Medicine, \\ Alexandria University, Alexandria, \\ Egypt \\ †Doctor Tamer Morsi passed away \\ on August 15, 2014
}

Correspondence: Hanaa Shafiek Chest Diseases Department, Faculty of Medicine, Alexandria University, El-Khartoom Square, Alexandria 21526, Egypt

Tel +20 I0 95907320

Email whitecoat_med@yahoo.com
Background: Bronchial vascular remodeling is an underresearched component of airway remodeling in COPD. Image-enhanced bronchoscopy may offer a less invasive method for studying bronchial microvasculature in COPD.

Objectives: To evaluate endobronchial mucosal vasculature and changes in COPD by imageenhanced i-scan3 bronchoscopy and correlate them pathologically by analyzing bronchial mucosal biopsies.

Methods: This case-control study analyzed 29 COPD patients (41.4\% Global initiative for chronic Obstructive Lung Disease B [GOLD B] and 58.6\% GOLD D) and ten healthy controls admitted at Alexandria Main University Hospital, Egypt. Combined high-definition white light bronchoscopy (HD WLB) with i-scan3 was used to evaluate endobronchial mucosal microvasculature. The vascularity was graded according to the level of mucosal red discoloration (ie, endobronchial erythema) from decreased discoloration to normal, mild, moderate, and severe increased red discoloration $(\mathrm{G}-1, \mathrm{G} 0, \mathrm{G}+1, \mathrm{G}+2$, and $\mathrm{G}+3$, respectively) and scored by three bronchoscopists independently. Bronchial mucosal biopsies were taken for microvascular density counting using anti-CD34 antibody as angiogenesis marker.

Results: Different grades of endobronchial erythema were observed across/within COPD patients using combined HD WLB + i-scan3, with significant agreement among scorers $(P=0.031$; median score of $\mathrm{G}+1[\mathrm{G}-1-\mathrm{G}+2])$ being higher in GOLD D $(P=0.001)$. Endobronchial erythema significantly correlated with COPD duration, exacerbation frequency, and body mass index $(P<0.05)$. Angiogenesis was significantly decreased among COPD patients versus controls (10.6 [8-13.3] vs 14 [11-17.1]; $P=0.02$ ). Mucosal surface changes (including edema, atrophy, and nodules) were better visualized by the combined HD WLB + i-scan3 rather than HD WLB alone.

Conclusion: Combined HD WLB + i-scan3 seems to be valuable in evaluating mucosal microvasculature and surface changes in COPD, which may represent vasodilatation rather than angiogenesis.

Keywords: COPD activity, endobronchial erythema, angiogenesis, vascular remodeling

\section{Introduction}

COPD is a heterogeneous disease characterized by various pathological structural changes (ie, remodeling). ${ }^{1}$ Bronchial vascular remodeling has been proposed to occur in COPD, although it seems to be less evident than in asthma, and may contribute to increased airway wall thickness and, therefore, may be associated with COPD progression. $^{2}$

The three main aspects of bronchial vascular remodeling are vasodilatation, increased microvascular permeability, and angiogenesis. ${ }^{3}$ The conventional method used to study bronchial vascular remodeling is immunohistochemical analysis of 
bronchial mucosal biopsy. Different markers of angiogenesis such as monoclonal antibodies against CD34, CD31, factor VIII, ${ }^{4}$ integrin avb3, ${ }^{5}$ and vascular endothelial growth factor were used for quantitative analysis of bronchial wall vascularity. ${ }^{3}$

Image-enhanced bronchoscopy may offer a less invasive technique to evaluate some aspects of bronchial vascularity. Early attempts have been made in the last 2 decades in order to study the endobronchial mucosal vasculature using highmagnification bronchovideoscopy to study subepithelial vessels of the bronchial mucosa. ${ }^{6,7}$ I-SCAN technology is the newly developed real-time image-enhancement endoscopy technology that is classified as a digital contrast method among endoscopic imaging techniques. ${ }^{8}$ I-SCAN technology combined with high definition has been widely used in gastroenterology ${ }^{9}$ and can unmask a plethora of small lesions. ${ }^{10}$

We hypothesized that I-SCAN bronchoscopy could evaluate endobronchial mucosal microvasculature in COPD patients in vivo. Accordingly, our objectives of the current study were to assess endobronchial vasculature and mucosal changes in COPD by image-enhanced bronchoscopy and to correlate them first pathologically by analyzing bronchial mucosal biopsies and second with lung function and evidence of COPD activity.

\section{Methods}

\section{Study design and population}

This was a prospective case-control study that invited 31 COPD patients diagnosed according to Global initiative for chronic Obstructive Lung Disease (GOLD) ${ }^{11}$ and 14 healthy non-COPD subjects to participate from January 2014 to February 2015 in Alexandria Main University Hospital, Egypt. COPD patients were clinically stable without evidence of exacerbation at the time of enrollment in the study. Patients with bronchial asthma, primary bronchiectasis, interstitial lung diseases, and lung cancer were excluded from the study. All subjects underwent bronchoscopy and subsequent bronchial mucosal biopsy was taken. The healthy subjects underwent fiberoptic bronchoscopy for a clinical indication of mainly foreign body extraction and alleged hemoptysis. However, two patients with COPD were excluded due to accidental diagnosis of lung cancer, whereas four healthy subjects were excluded due to inadequate processing of bronchial mucosal biopsies. Accordingly, 29 COPD patients and ten healthy controls were analyzed. The study protocol was approved by the local ethical committee of Alexandria University, Egypt and all the participants signed informed consent.

\section{Characteristics of the patients}

The following clinical history data of patients were collected: frequency of acute exacerbation of COPD (AECOPD) in the last year as well as forced spirometry. COPD assessment test score, GOLD score, ${ }^{11}$ and Body mass index, Airflow obstruction, Dyspnea, and Exercise capacity (BODE) index ${ }^{12}$ were used for further assessing COPD patients. Chest computed tomography scan was performed for all patients to evaluate both airways and parenchyma. Apical bronchus of the right upper lobe was used for bronchial wall thickness measurement by different ways ${ }^{13,14}$ (Figure 1) from axial computed tomography images with the use of inspiratory acquisitions with digital imaging and communications in medicine (DICOM) software (OsiriX DICOM Viewer, Version 4.0, 32-bit; Pixmeo Medical Imaging Software Development Company, Geneva, Switzerland). Emphysema was scored visually according to the modified Goddard scoring system. ${ }^{15}$ Level of $-950 \mathrm{HU}$ to $-1,020 \mathrm{HU}$ was chosen to describe emphysematous changes in the lungs. ${ }^{16}$

\section{Bronchoscopy}

High-definition white light bronchoscopy (HD WLB; Pentax, EB-1970TK with Pentax EP-i5000K video processor, Tokyo, Japan) was performed under conscious sedation using midazolam 3-7 mg. Image-enhanced i-scan3 visualization (surface-contrast-tone enhancement) was then turned on and added to the HD WLB for reevaluation of the mucosal surface texture and blood vessels of the bronchial tree. i-scan 3 settings were: brightness average, redness 0 , blueness 0 , enhancement level +2 , surface enhancement +3 , tone enhancement gastric, color enhancement off, and noise reduction low. Images of the bronchial wall of the main, lobar, and distal bronchi were continuously recorded for later off-line analysis.

\section{Image analysis}

All the recorded images and videos of both HD WLB and i-scan3 were analyzed visually by three independent bronchoscopists. A subjective score was developed for evaluation of vascular changes. This score depended on i-scan3 property in reconstruction of virtual images in real time by increasing the intensity of narrowed blue light to maximum and by decreasing narrowed red light and green light to minimum resulting in an improved contrast of the capillary patterns and enhancement of the mucosal surface. ${ }^{9}$ We classified the grade of erythema - using combined HD WLB + i-scan 3 - according to the level of mucosal red discoloration enhancement against bluish background ranging from grade 

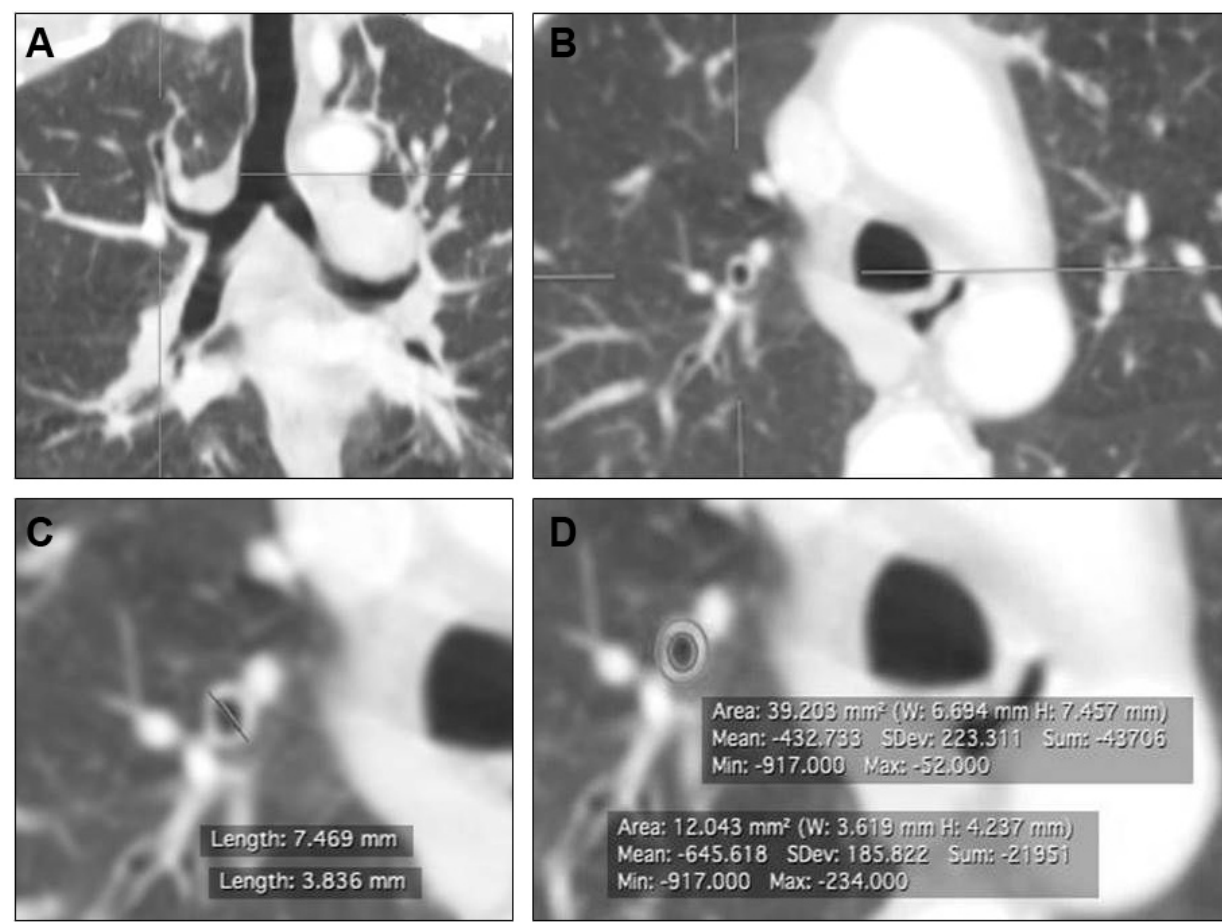

Figure I Bronchial wall thickness measurement in high-resolution CT scan.

Notes: Coronal (A) and axial (B) cuts demonstrated the identification of apical segmental bronchus of right upper lobe bronchus (RBI). (C) Shows axial cut of chest $\mathrm{CT}$ showing circumferential measures of outer and inner areas of RBI to calculate airway cross-sectional area (PWA\%). (D) Shows axial cut of chest CT measuring the inner and outer diameters to calculate $\mathrm{I} / \mathrm{O}$ ratio and T/D ratio ([inner diameter - outer diameter/2] to outer bronchus diameter).

Abbreviations: CT, computed tomography; I/O ratio, inner to outer diameter ratio; SD, standard deviation; W, width; H; height; Min, minimum; Max, maximum; T/D ratio, bronchial wall thickness to outer diameter ratio.

(G) -1 to G+3. Grade 0 reflects normal bronchial mucosa vasculature detected in healthy controls, $\mathrm{G}-1$ reflects decreased mucosal vascularity, while $\mathrm{G}+1$ (mild), $\mathrm{G}+2$ (moderate), and $\mathrm{G}+3$ (severe) reflect increased mucosal red discoloration. In case of heterogeneous endobronchial erythema in the tracheobronchial tree of the same patient, the grade was evaluated using visual mean. Furthermore, bronchial mucosal surface changes including mucosal nodules, edema, atrophy, striations, and stoma were described. The detected bronchial mucosal and vascular changes in COPD were compared with normal findings of controls. The generated grading system was evaluated by three bronchoscopists independently, and agreement of their readings' median was tested.

\section{Pathological evaluation}

Bronchial mucosal biopsies were taken from accessible bronchi with no specific predetermined location as the changes found were diffuse. The biopsies were immediately fixed in $10 \%$ formalin solution overnight, dehydrated in graded series of ethanol, and then embedded in paraffin. Serial $4 \mu \mathrm{m}$ thick sections were cut, dewaxed, and stained by hematoxylin and eosin for routine histological examination followed by immunohistochemical staining using anti-CD34 monoclonal antibody (\#MA5-18091 at a dilution of $1 \mu \mathrm{g} / \mathrm{mL}$; Thermo Fisher Scientific, Waltham, MA, USA) to highlight the endothelial cells of newly formed blood vessels. Angiogenesis was evaluated by manual semiquantitative counting of microvessels highlighted using anti-CD34 antibody in ten high power fields as a measure of microvascular density (MVD).

\section{Statistical analysis}

All the data were presented as median and interquartile range (IQR $=25$ th-75th percentile) or number and percentage (\%). The quantitative variables were not normally distributed by Kolmogorov-Smirnov test. Comparisons between groups were performed using Mann-Whitney $U$, Fisher's exact, or Monte Carlo significance tests as appropriate and Spearman rank correlation test. The receiver operating characteristic curve and area under the curve analysis were used to test the accuracy, sensitivity, and specificity of the diagnostic utility of CD34. Agreement between investigators regarding I-SCAN score readings was performed by two-way mixed effect model intraclass correlation (ICC), where people effects are random and measure effects are fixed. Data were analyzed using the Statistical Package for Social Sciences 
(SPSS Version 20; IBM Corporation, Armonk, NY, USA) and $P$-value $\leq 0.05$ using two-sided test was considered as significant.

\section{Results}

\section{Study population}

The baseline characteristics of both the control and the COPD patients are shown in Table 1 . The COPD patients were highly symptomatic with a median COPD assessment test score of 13 and ranging from 10 to 21 and moderate to severe airway obstruction. Accordingly, 41.4\% (12 patients) were GOLD B and 58.6\% (17 patients) were GOLD D (Table 1). The clinical and functional characteristics of COPD patients according to GOLD score are shown in Table 2. The duration of COPD, AECOPD frequency, and BODE index were significantly higher in GOLD D versus GOLD B $(P<0.05)$. Bronchial wall thickness was detected in all patients without statistically significant difference according to GOLD score $(P>0.05$; Table 2).

\section{Vascular changes}

Different grades of endobronchial erythema were observed using combined HD WLB and i-scan3 across COPD patients (Figure 2) as well as within the same endobronchial tree of some patients, ie, heterogeneous endobronchial erythema with significant agreement among the three bronchoscopists (ICC $=0.133,95 \%$ confidence interval $[\mathrm{CI}]:-0.024$ to 0.347 , $P=0.031)$ as well as among two independent readings of one bronchoscopist (ICC $=0.239,95 \% \mathrm{CI}$ : -0.096 to 0.548 , $P=0.005)$. The median grade of the bronchoscopists was $\mathrm{G}+1$ $(\mathrm{IQR}=\mathrm{G}-1-\mathrm{G}+2)$ for the COPD patients being significantly higher in GOLD D versus GOLD B (G+1 [IQR = G-1-G+2] vs G0 $[\mathrm{IQR}=\mathrm{G}-1-\mathrm{G}+1] ; P=0.001)$. However, the median grade reading of the bronchoscopists for the healthy control was G0 (IQR = G-1-G+1) without statistical significant difference compared with the COPD group $(P=0.379)$.

\section{Endobronchial mucosal changes}

Different mucosal changes were clearly identified by highdefinition i-scan3 bronchoscopy better than HD WLB in COPD patients (Figure 3). Mucosal thinning was the most common mucosal change that was detected in $92 \%$, while mucosal folds and edema were present in $65.3 \%$ of the patients. Mucosal stoma and nodules were observed in $30 \%$ and $38.4 \%$, respectively. Tracheomalacia was detected during bronchoscopic session in 19\%.

\section{Angiogenesis assessment}

Histopathological examination of bronchial mucosal biopsies revealed chronic inflammatory changes. COPD patients had significantly lower angiogenesis (MVD) in comparison with controls (10.6 [8.65-13.2] vs 14.25 [11-15.2] respectively, $P=0.02$; Figure $4 \mathrm{~A}-\mathrm{C})$. However, there was no significant difference between GOLD B and GOLD D $(P=0.826)$. By receiver operating characteristic curve analysis, a cutoff MVD of $\leq 13.7$ had the sensitivity of $81.5 \%$ and specificity of $55.6 \%$ to discriminate COPD patients from healthy controls with a diagnostic accuracy of $75.7 \%$ (area under the curve $=$ $0.757,95 \% \mathrm{CI}:=0.559-0.866, P=0.022$; Figure 4D).

\section{Correlations}

Grade of endobronchial erythema detected by i-scan3 correlated positively with duration of COPD, frequency of AECOPD, and body mass index (BMI; $r=0.4, P=0.02$; $r=0.1, P=0.004$ and $r=0.3, P=0.03$, respectively) but not

Table I Baseline characteristics of the study population

\begin{tabular}{|c|c|c|c|}
\hline Variable & COPD (n=29) & Controls $(n=10)$ & $\begin{array}{l}\text { Significant } \\
\text { (P-value) }\end{array}$ \\
\hline Age (years) & $60(53-65)$ & $30.5(20.8-52.3)$ & $0.001 *$ \\
\hline \multicolumn{4}{|l|}{ Sex } \\
\hline Male/female; n (\%) & $21(72.4) / 8(27.6)$ & $5(50) / 5(50)$ & 0.250 \\
\hline \multicolumn{4}{|l|}{ Smoking history } \\
\hline Current/former/passive; n (\%) & $16(55.2) / 5(17.2) / 8(27.6)$ & $5(50) / 0(0) / 5(50)$ & 0.290 \\
\hline Pack/year index & $57.5(35-75)$ & $30(24-35)$ & $0.047 *$ \\
\hline \multicolumn{4}{|l|}{ Spirometry } \\
\hline $\mathrm{FEV} / \mathrm{FVC}$ & $55(46-60)$ & $82(77.8-88.3)$ & $0.00 I^{*}$ \\
\hline $\mathrm{FEV}_{1}(\mathrm{~L})$ & $1.4(0.8-1.75)$ & $2.75(2.5-3.1)$ & $0.002 *$ \\
\hline $\mathrm{FEV} \%$ predicted & $54(35-65)$ & $90(72.5-106)$ & $0.008^{*}$ \\
\hline \multicolumn{4}{|l|}{ GOLD stage } \\
\hline GOLD B/GOLD D & $12(4 \mid .4) / 17(58.6)$ & - & NA \\
\hline
\end{tabular}

Note: $* P \leq 0.05$ is considered significant. Data expressed as median (IQR=25th-75th percentile) unless otherwise stated. “-” not relevant.

Abbreviations: $\mathrm{FEV}_{1}$, forced expiratory volume I; FVC, forced vital capacity; GOLD, Global initiative for chronic Obstructive Lung Disease; IQR, interquartile range; NA, not assessed. 
Table 2 Clinical and functional characteristics of COPD according to GOLD scores

\begin{tabular}{|c|c|c|c|}
\hline Variable & $\begin{array}{l}\text { GOLD B } \\
(n=12 ; 41.4 \%)\end{array}$ & $\begin{array}{l}\text { GOLD D } \\
(n=17 ; 58.6 \%)\end{array}$ & $\begin{array}{l}\text { Significant } \\
P \text {-value }\end{array}$ \\
\hline Age (years) & $62(60-67.5)$ & $58(50.8-63.5)$ & 0.2 \\
\hline \multicolumn{4}{|l|}{ Sex; n (\%) } \\
\hline Male/female & $10(83.3) / 2(16.7)$ & II (64.7)/6 (35.3) & 0.6 \\
\hline \multicolumn{4}{|l|}{ Smoking history; n (\%) } \\
\hline Current/former/passive & $7(58.3) / 3(25) / 2(16.7)$ & $9(53) / 2(11.7) / 6(35.3)$ & 0.441 \\
\hline Pack year index & $50(33.8-70)$ & $60(28.8-80)$ & 0.9 \\
\hline BMI $\left(\mathrm{kg} / \mathrm{m}^{2}\right)$ & $24.2(20.4-27.4)$ & $25.6(23.98-28.7)$ & 0.1 \\
\hline 6MWT (m) & $212.5(200-220)$ & $200(180-216.3)$ & 0.4 \\
\hline MMRC dyspnea scale & $I(I-I .5)$ & $2(1-2.25)$ & 0.05 \\
\hline CAT score & $12(12-14)$ & $14(12-16)$ & 0.19 \\
\hline COPD duration (years) & $5(3.5-10)$ & $12(5.75-20)$ & $0.007^{*}$ \\
\hline AECOPD frequency/year & $I(I-I)$ & $2(2-2)$ & $<0.00 I^{*}$ \\
\hline Comorbidities; n (\%) & $3(27)$ & $9(50)$ & 0.27 \\
\hline BODE index & $3(2.5-4)$ & $5(3-6.3)$ & $0.04 *$ \\
\hline \multicolumn{4}{|l|}{ Spirometry } \\
\hline $\mathrm{FEV}_{1} / \mathrm{FVC}$ & $60(52.5-67.5)$ & $48(39-59)$ & $0.005^{*}$ \\
\hline $\mathrm{FEV}_{1}(\mathrm{~L})$ & $1.76(1.58-1.98)$ & $0.995(0.75-1.4)$ & $0.00 I^{*}$ \\
\hline $\mathrm{FEV}_{1} \%$ & $62(54.5-72.5)$ & $40(25.5-60)$ & $<0.00 I^{*}$ \\
\hline $\mathrm{FEF}_{25-75} \%$ & $53(40.5-83.5)$ & $16.5(9.5-42)$ & $0.0 I^{*}$ \\
\hline \multicolumn{4}{|l|}{ Chest CT scan } \\
\hline \multicolumn{4}{|l|}{ Bronchial wall thickness } \\
\hline PWA\% & 75 (74-78) & $77(76-80)$ & 0.4 \\
\hline T/D ratio & $0.26(0.24-0.29)$ & $0.26(0.23-0.3)$ & 0.9 \\
\hline I/O ratio & $0.49(0.4-0.5 \mathrm{I})$ & $0.44(0.39-0.5)$ & 0.2 \\
\hline Emphysematous changes; n (\%) & $8(66.7)$ & $12(70.6)$ & \\
\hline Emphysema visual score & I $(0.5-2)$ & I $(0.5-2)$ & 0.8 \\
\hline \multicolumn{4}{|l|}{ Emphysema distribution; n (\%) } \\
\hline Upper lobe & $5(57.1)$ & $4(38.5)$ & \\
\hline Upper + lower lobes & I (I4.3) & $5(38.5)$ & 0.59 \\
\hline Lower lobe & $2(28.6)$ & $3(23.1)$ & \\
\hline
\end{tabular}

Notes: PWA: airway cross-sectional area (PWA $>63 \%$ indicated bronchial wall thickness ${ }^{13}$ ), T/D ratio: (inner diameter - outer diameter/2) to outer bronchus diameter (T/D ratio $>0.22$ indicated bronchial wall thickness ${ }^{14}$ ), $1 / O$ ratio $<0.8$ indicated bronchial wall thickness, ${ }^{13} * P \leq 0.05$ is considered significant. Data expressed as median (IQR=25th-75th percentile) unless otherwise stated.

Abbreviations: GOLD, Global initiative for chronic Obstructive Lung Disease; BMI, body mass index; 6MWT, 6-minute walk test; CAT, COPD assessment test; AECOPD, acute exacerbation of COPD; FEV , forced expiratory volume after I second; FVC, forced vital capacity; FEF, forced expiratory flow; CT, computed tomography; I/O ratio, inner to outer diameter ratio; BODE, Body mass index, Airflow obstruction, Dyspnea, and Exercise capacity index; PWA, percentage of airway cross-sectional area; T/D ratio, bronchial wall thickness to outer diameter ratio; IQR, interquartile range; MMRC, modified Medical Research Council scale.

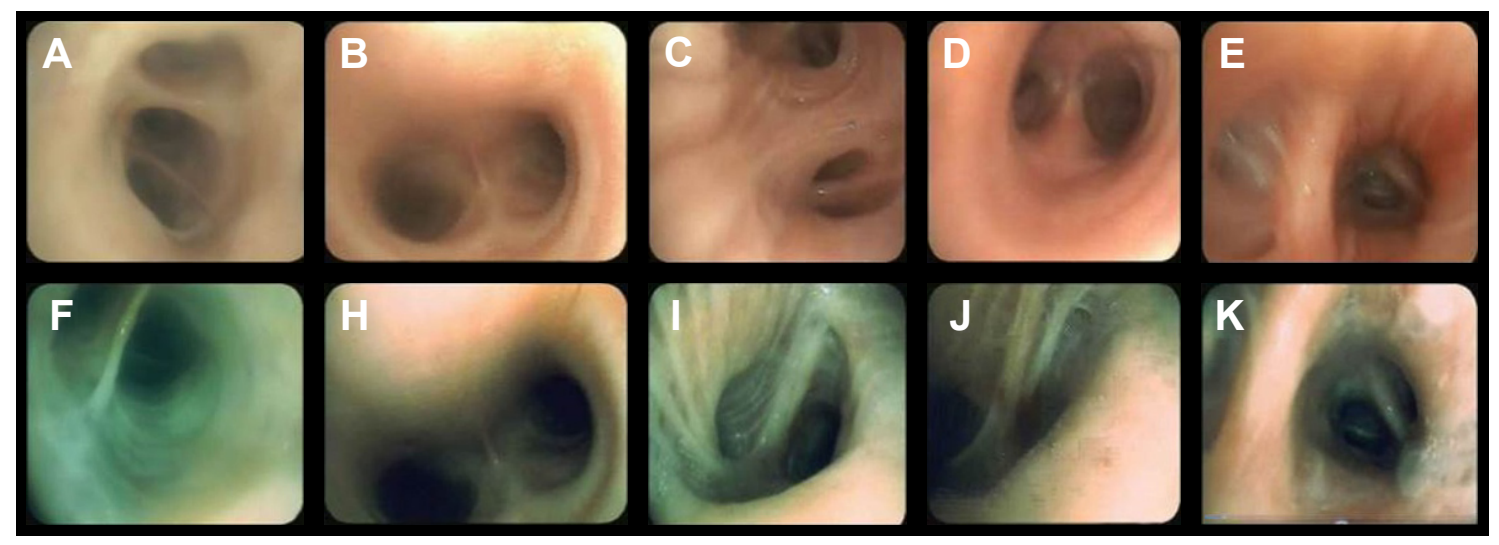

Figure 2 The different grades of endobronchial erythema identified by HD WLB (A-E) and i-scan3 (F, H-K).

Notes: $\mathbf{A}$ and $\mathbf{F}$ show decreased erythema $(\mathbf{H}-\mathbf{J}) ; \mathbf{B}$ and $\mathbf{H}$ show normal erythema $(\mathrm{G} 0)$; $\mathbf{C}$ and $\mathbf{I}$ show mild erythema $(\mathrm{G}+\mathrm{I})$; $\mathbf{D}$ and $\mathbf{J}$ show moderate erythema (G+2); and $\mathbf{E}$ and $\mathbf{K}$ show severe erythema $(\mathrm{G}+3)$.

Abbreviations: HD WLB, high-definition white light bronchoscopy; G, grade of erythema. 


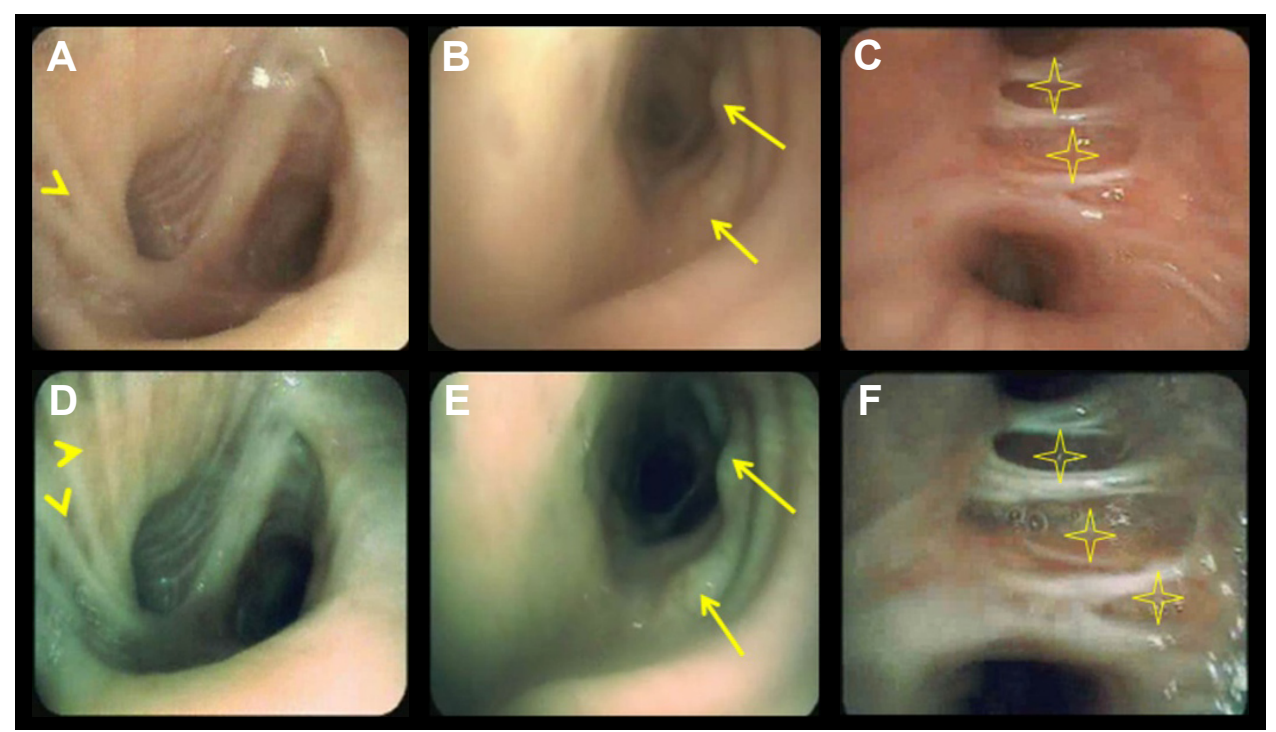

Figure 3 HD WLB (A-C) and i-scan3 (D-F) showing various endobronchial mucosal changes.

Notes: $\mathbf{A}$ and $\mathbf{D}$ show mucosal striations (arrowheads), edema, and stoma; $\mathbf{B}$ and $\mathbf{E}$ show mucosal nodules (arrows); and $\mathbf{C}$ and $\mathbf{F}$ show mucosal thinning (stars). Abbreviation: HD WLB, high-definition white light bronchoscopy.

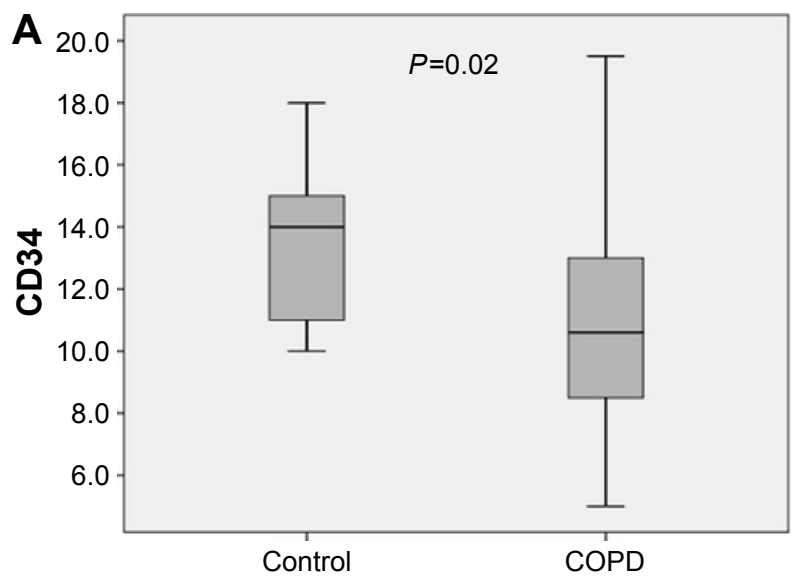

B
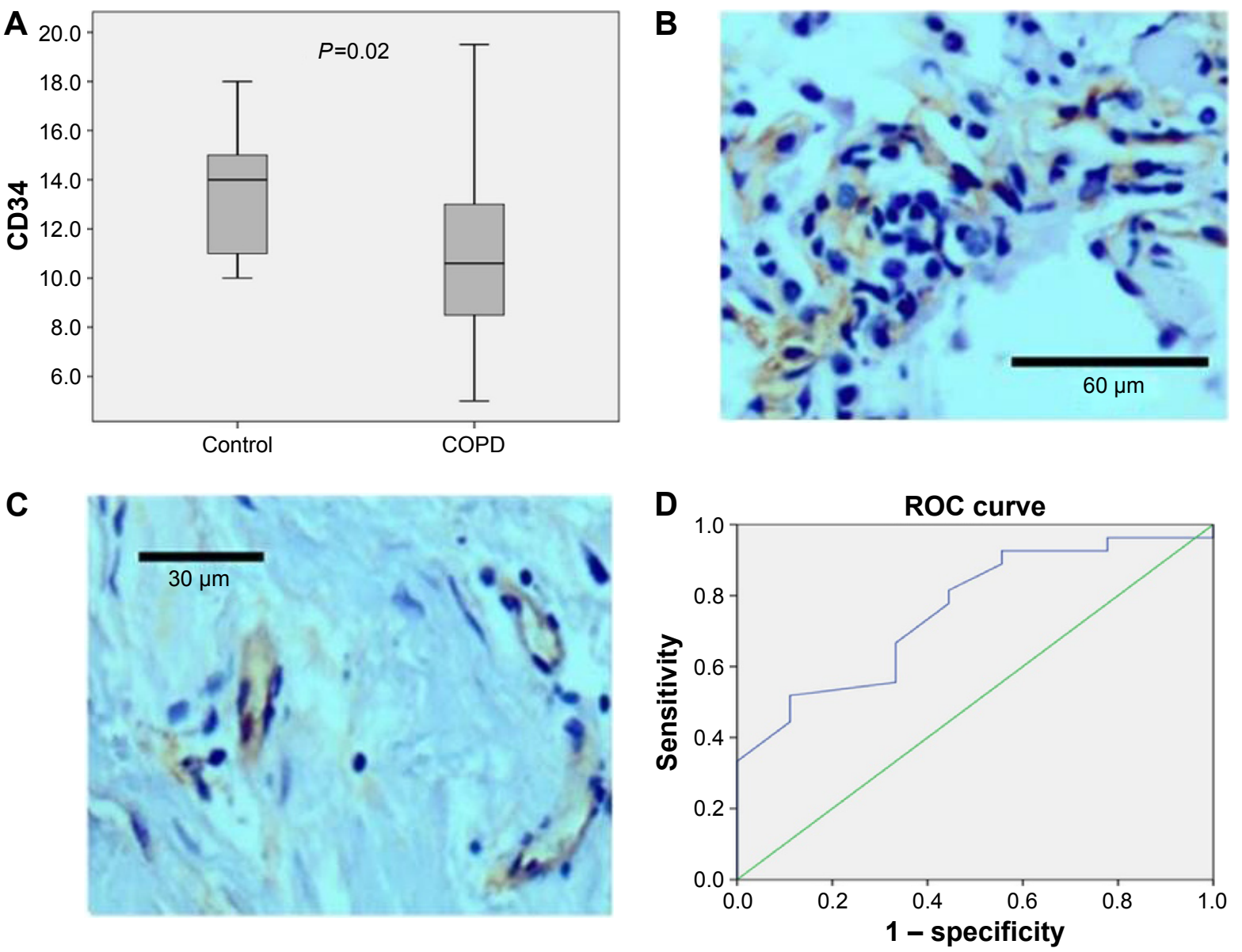

Figure 4 Endobronchial mucosal microvasculature in COPD and controls.

Notes: (A) Distribution of CD34 among COPD patients and healthy controls; (B) a section through the bronchial lamina propria shows high microvascular density in a control subject (anti-CD34, $\times 400$ ); (C) shows low microvascular density in a COPD patient as highlighted by CD34 endothelial immunostaining (anti-CD34, $\times 400$ ); and (D) ROC curve to evaluate angiogenesis between COPD patients and healthy controls.

Abbreviation: ROC, receiver operating characteristic. 
with BODE index and pulmonary function parameters $(P>0.05)$. However, MVD inversely correlated only with aging ( $r=-0.38, P=0.04)$.

Mucosal nodules and edema detected by i-scan3 were significantly associated with active smoking status $(P=0.02$ and 0.05 , respectively), while mucosal atrophy was significantly associated with cumulative smoking index $(P=0.02)$ and tracheomalacia with spirometric grade of airflow obstruction $(P=0.03)$.

Moreover, we observed positive correlation between MVD and bronchial wall thickness measured radiologically by airway cross-sectional area $(r=0.48, P=0.01)$, while endobronchial erythema by i-scan3 was significantly associated with mucosal atrophy $(P=0.047)$ and negatively correlated with bronchial wall thickness despite being insignificant ( $r=-0.37, P=0.07$ ).

\section{Discussion}

To our knowledge, this is the first study to use combined HD WLB and i-scan3 image enhancement to study the microvascular abnormalities of the airways in COPD patients in vivo that are confirmed by histopathologically and correlate them with both radiological and lung function measurements. It also shows that this combined bronchoscopic technique can delineate the different endobronchial mucosal changes - which represent another limb of bronchial wall remodeling - in this group of patients better than HD WLB alone. We believe that these results highlighted the vascular remodeling in COPD that could be a target for future therapeutic interventions.

\section{Previous studies}

Thompson et $\mathrm{al}^{17}$ described the bronchitis index after examination of the endobronchial mucosa by bronchoscopy using a scale that grades erythema, edema, and friability of the mucosa as well as secretions. They found that this index had no correlation with spirometric values within COPD patients, which may suggest that elevation of bronchitis index is not a function of airway obstruction. Hashimoto et $\mathrm{al}^{18}$ and Calabrese et $\mathrm{al}^{19}$ found that there was no correlation between the degree of vascularity, vascular area, or vessel size and airflow limitation in COPD patients, and they suggested that angiogenesis seems independent of the development of airway obstruction and not related to its severity. Our current results agree with these findings.

\section{Interpretation of results}

Combined HD WLB and i-scan3 image enhancement was able to identify different degrees of endobronchial mucosal erythema across and within COPD patients with significant agreement among scorers. Thus highlighting the concept that COPD is a heterogeneous disease across individuals and regionally in the lung. Tanaka et al ${ }^{7}$ visualized blood microvessels on bronchial mucosa using a high-magnification bronchovideoscope in subjects with COPD. Recently, van der Heijden et al ${ }^{20}$ showed that HD WLB and I-SCAN bronchoscopy were significantly better for the detection of subtle vascular abnormalities in the airways compared with autofluorescence videobronchoscopy in case of suspicious preinvasive malignant lesions.

Furthermore, endobronchial mucosal erythema was significantly higher in severe GOLD score, ie, GOLD D and significantly correlated with the duration of the disease as well as the frequency of AECOPD and BMI. COPD is well known as a heterogeneous multicomponent disease that is associated with vascular abnormalities ${ }^{21}$ and that the frequency of AECOPD and BMI are considered as markers of COPD progression and activity. ${ }^{22}$ The vascular component of airway remodeling is an important contributor that may alter management strategy of COPD. ${ }^{2}$ Ikeda et $\mathrm{al}^{23}$ reported that the presence of various endobronchial mucosal changes such as erythema, edema, secretions, and atrophic mucosa may give evidence of active inflammation. Accordingly, we may suggest that grade of endobronchial erythema may be a marker of COPD activity.

Moreover, we found that COPD patients had significantly lower angiogenesis as being identified by CD34 compared with healthy controls despite the higher endobronchial erythema grade in COPD patients, and that low MVD counting by CD34 discriminated between COPD and controls with diagnostic accuracy of $75.7 \%$ and sensitivity of $81 \%$. This could be explained on the basis of many factors. First, increased vascular area, ie, vasodilation and vascular leakage of both large and small airway wall are the dominant aspects of bronchial vascular remodeling in COPD rather than new vessel formation, ${ }^{18,19,24,25}$ while angiogenesis and vascular leakage are more prevalent in asthma.,24 Second, aging - which is considered as one of the risk factors for the development of COPD ${ }^{11}$ - could be a cause of decreased angiogenesis, ${ }^{26}$ as we found a significant inverse correlation between these two variables. Interestingly, we observed positive correlation between bronchial angiogenesis and bronchial wall thickness denoting increased proportion of the wall occupied by newly formed vessels. However, angiogenesis as previously observed in this study and others has not been reported as a particular feature of COPD in contrast to asthma. ${ }^{27}$ 


\section{Clinical implications}

Our pilot descriptive study pinpoints to the effectiveness of combined HD WLB and i-scan3 bronchoscopy as minimally invasive tool for in vivo assessment of endobronchial mucosal microvascular remodeling as a marker of activity in COPD patients that should be considered in future therapeutic interventions, and that this tool can help in vivo tracking the effects of various therapeutic interventions for COPD. However, further studies are needed for clearer understanding of angiogenesis and its role in COPD pathogenesis that may lead to the development of selective molecules with antiangiogenic properties and fewer side effects, which may be beneficial for this group of patients. ${ }^{28}$ Chetta and Olivieri ${ }^{2}$ reported that in COPD, there is less experimental evidence on the effect of inhaled steroids on airway microvascular changes, in contrast to asthma where inhaled steroids can downregulate vascular remodeling in asthmatic airways by acting on proangiogenic factors.

\section{Limitations of the study}

The present study has some limitations. First, we did not use other markers of angiogenesis, which have been approved to be related to pathophysiology of COPD.$^{29}$ However, CD34 is considered an acceptable highlighter for endothelial cells of blood vessels in order to count the MVD. ${ }^{30}$ Second, we did not incorporate other bronchoscopic techniques such as the narrow band bronchoscopic imaging or autofluorescence bronchoscopy in the current study. However, in the literature these techniques are used mainly for early detection of lung cancer rather than studying airway diseases. ${ }^{31}$

\section{Conclusion}

Combined image enhancing i-scan3 with HD WLB seems to be valuable in evaluating endobronchial mucosal microvasculature in COPD and better in detecting mucosal surface changes rather than HD WLB alone. Furthermore, endobronchial erythema visualized by bronchoscopy may represent a measure of COPD activity and an indicator of bronchial vasodilation and vascular leakage rather than angiogenesis that is significantly decreased among COPD patients. These findings could modify disease management and prognosis.

\section{Disclosure}

The authors report no conflicts of interest in this work.

\section{References}

1. Sköld CM. Remodeling in asthma and COPD - differences and similarities. Clin Respir J. 2010;4(suppl 1):20-27.
2. Chetta A, Olivieri D. Role of inhaled steroids in vascular airway remodelling in asthma and COPD. Int J Endocrinol. 2012;2012:397693.

3. Feltis BN, Wignarajah D, Zheng L, et al. Increased vascular endothelial growth factor and receptors: relationship to angiogenesis in asthma. $\mathrm{Am}$ J Respir Crit Care Med. 2006;173(11):1201-1207.

4. Müller AM, Hermanns MI, Skrzynski C, et al. Expression of the endothelial markers PECAM-1, vWf, and CD34 in vivo and in vitro. Exp Mol Pathol. 2002;72(3):221-229.

5. Brooks PC, Clark RA, Cheresh DA. Requirement of vascular integrin alpha $v$ beta 3 for angiogenesis. Science. 1994;264(5158):569-571.

6. Shibuya K, Hoshino H, Chiyo M, et al. Subepithelial vascular patterns in bronchial dysplasias using a high magnification bronchovideoscope. Thorax. 2002;57(10):902-907.

7. Tanaka H, Yamada G, Saikai T, et al. Increased airway vascularity in newly diagnosed asthma using a high-magnification bronchovideoscope. Am J Respir Crit Care Med. 2003;168(12):1495-1499.

8. Tajiri H, Niwa H. Proposal for a consensus terminology in endoscopy: how should different endoscopic imaging techniques be grouped and defined? Endoscopy. 2008;40:775-778.

9. Kodashima S, Fujishiro M. Novel image-enhanced endoscopy with i-scan technology. World J Gastroenterol. 2010;16(9):1043-1049.

10. Hoffman A, Basting N, Goetz M, et al. High-definition endoscopy with i-Scan and Lugol's solution for more precise detection of mucosal breaks in patients with reflux symptoms. Endoscopy. 2009;41(2):107-112.

11. Vestbo J, Hurd SS, Agustí AG, et al. Global strategy for the diagnosis, management, and prevention of chronic obstructive pulmonary disease: GOLD executive summary. Am J Respir Crit Care Med. 2013;187(4): 347-365.

12. Celli BR, Cote CG, Marin JM, et al. The body-mass index, airflow obstruction, dyspnea, and exercise capacity index in chronic obstructive pulmonary disease. $N$ Engl J Med. 2004;350(10):1005-1012.

13. Nakano Y, Muro S, Sakai H, et al. Computed tomographic measurements of airway dimensions and emphysema in smokers. Correlation with lung function. Am J Respir Crit Care Med. 2000;162(3 pt 1): 1102-1108.

14. Kim SS, Seo JB, Lee HY, et al. Chronic obstructive pulmonary disease: lobe-based visual assessment of volumetric CT by Using standard images - comparison with quantitative CT and pulmonary function test in the COPDGene study. Radiology. 2013;266(2):626-635.

15. Goddard PR, Nicholson EM, Laszlo G, Watt I. Computed tomography in pulmonary emphysema. Clin Radiol. 1982;33(4):379-387.

16. Wang Z, Gu S, Leader JK, et al. Optimal threshold in CT quantification of emphysema. Eur Radiol. 2013;23(4):975-984.

17. Thompson AB, Huerta G, Robbins RA, et al. THe bronchitis index. A semiquantitative visual scale for the assessment of airways inflammation. Chest. 1993;103(5):1482-1488.

18. Hashimoto M, Tanaka H, Abe S. Quantitative analysis of bronchial wall vascularity in the medium and small airways of patients with asthma and COPD. Chest. 2005;127(3):965-972.

19. Calabrese C, Bocchino V, Vatrella A, et al. Evidence of angiogenesis in bronchial biopsies of smokers with and without airway obstruction. Respir Med. 2006;100(8):1415-1422.

20. van der Heijden EH, Hoefsloot W, van Hees HW, Schuurbiers OC. High definition bronchoscopy: a randomized exploratory study of diagnostic value compared to standard white light bronchoscopy and autofluorescence bronchoscopy. Respir Res. 2015;16:33.

21. Santos S, Peinado VI, Ramírez J, et al. Characterization of pulmonary vascular remodelling in smokers and patients with mild COPD. Eur Respir J. 2002;19(4):632-638.

22. Agusti A, MacNee W. The COPD control panel: towards personalised medicine in COPD. Thorax. 2013;68(7):687-690.

23. Ikeda S, Yanai N, Ishikawa S. Flexible bronchofiberscope. Keio J Med. 1968;17:1-16.

24. Zanini A, Chetta A, Imperatori AS, Spanevello A, Olivieri D. The role of the bronchial microvasculature in the airway remodelling in asthma and COPD. Respir Res. 2010;11:132. 
25. Zanini A, Chetta A, Saetta M, et al. Bronchial vascular remodelling in patients with COPD and its relationship with inhaled steroid treatment Thorax. 2009;64(12):1019-1024.

26. Meyer KC, Cardoni AL, Xiang Z, Cornwell RD, Love RB. Vascular endothelial growth factor in human lung transplantation. Chest. 2001;119(1):137-143.

27. Kuwano K, Bosken CH, Paré PD, Bai TR, Wiggs BR, Hogg JC. Small airways dimensions in asthma and in chronic obstructive pulmonary disease. Am Rev Respir Dis. 1993;148(5):1220-1225.

28. Paredi P, Barnes PJ. The airway vasculature: recent advances and clinical implications. Thorax. 2009;64(5):444-450.
29. Carmeliet P. Mechanisms of angiogenesis and arteriogenesis. Nat Med. 2000;6(4):389-395.

30. Traweek ST, Kandalaft PL, Mehta P, Battifora H. The human hematopoietic progenitor cell antigen (CD34) in vascular neoplasia. Am J Clin Pathol. 1991;96(1):25-31.

31. Zaric B, Stojsic V, Sarcev T, et al. Advanced bronchoscopic techniques in diagnosis and staging of lung cancer. J Thorac Dis. 2013;5(suppl 4): S359-S370.

International Journal of COPD

\section{Publish your work in this journal}

The International Journal of COPD is an international, peer-reviewed journal of therapeutics and pharmacology focusing on concise rapid reporting of clinical studies and reviews in COPD. Special focus is given to the pathophysiological processes underlying the disease, intervention programs, patient focused education, and self management protocols.

\section{Dovepress}

This journal is indexed on PubMed Central, MedLine and CAS. The manuscript management system is completely online and includes a very quick and fair peer-review system, which is all easy to use. Visit http://www.dovepress.com/testimonials.php to read real quotes from published authors.

Submit your manuscript here: http://www.dovepress.com/international-journal-of-chronic-obstructive-pulmonary-disease-journal 